\title{
Kyle equilibrium under random price pressure.*
}

\author{
José Manuel Corcuera ${ }^{\dagger} \quad$ Giulia Di Nunno $; \quad$ José Fajardo ${ }^{\S}$
}

November 18, 2018

\begin{abstract}
We study the equilibrium in the model proposed by Kyle in 1985 and extended to the continuous time setting by Back in 1992. The novelty of this paper is that we consider a framework where the price pressure can be random. We also allow for a random release time of the fundamental value of the asset. This framework includes all the particular Kyle models proposed in the literature. The results enlighten the equilibrium properties shared by all these models and guide the way of finding equilibria in this context.
\end{abstract}

Key words: Kyle model, market microstructure, equilibrium, insider trading, stochastic control, semimartingales, enlargement of filtrations.

JEL-Classification C61· D43· D44· D53. G11· G12· G14

MS-Classification 2010: 60G35, 62M20, 93E10, 94Axx

\section{Introduction}

In mathematical finance there are different kind of models to treat the effect of privilege information or informational asymmetries. In some models the prices are fixed exogenously, i.e. the insider does not affect the stock price dynamics, and the privileged information is a functional of the stock price process, e.g., the maximum, the final value, etc. This is the case of Karatzas and Pikovsky (1996), Amendiger et al. (1998), Grorud and Pontier (1998, 2001, 2005), Imkeller et al. (2001), Corcuera et al. (2004), Biagini and Øksendal

\footnotetext{
${ }^{*}$ Acknowledgement. The work of J.M. Corcuera is supported by the Spanish grant MTM2013-40782-P; G. Di Nunno acknowledges the financial support of the Research Council of Norway (RCN) as this research is carried out within STORM: Stochastics for Time-Space Risk Models, project 274410; J. Fajardo thanks financial support from CNPq-Brazil grant $\mathrm{n}^{\circ}$ 302693/2017-3.

$\dagger$ Universitat de Barcelona, Gran Via de les Corts Catalanes, 585, E-08007 Barcelona, Spain. E-mail: jmcorcuera@ub.edu.

${ }^{\ddagger}$ University of Oslo, Centre of Mathematics for Applications, P.O. Box 1053 Blindern NO-0316 Oslo, and NHH, Institutt for Foretaks Økonomi, Helleveien 30, 5045 Bergen, Norway. E-mail: giulian@math.uio.no

$\S$ Brazilian School of Public and Business Administration, Getulio Vargas Foundation. Rua Jornalista Orlando Dantas 30 , 22231-010, Rio de Janeiro. RJ-Brazil. E-mail:jose.fajardo@fgv.br.
} 
(2005, 2006), Kohatsu-Higa (2007), Di Nunno et al. (2006, 2011), Draouil and Øksendal (2016), Ernrst et al. (2017) and the references therein. In some other models, the impact of the insider strategy affects the stock price dynamics and the insider becomes a large trader as well. This is the case of Di Nunno et al. (2008) (where the authors talk about a large insider) and Kohatsu-Higa (2007). But if market prices are determined by the demand of the market participants the privileged information cannot be a functional of the stock price process because this would imply the very knowledge of the future demand and that is, in our opinion, difficult to interpret. This comment does not include models with large traders like, for instance the paper of Cuoco and Cvitanic (1998), in the sense that it is realistic to assume that the dynamics of the stock is affected by large investments. Neither it includes when the privileged information is exogenously given, through the knowledge of the value of the fundamental price, or some signal of it, or the announcement time of the release of the fundamental price, which evolves independently of the demand.

We consider this latter situation. The questions addressed in this paper deal with the characterization of the equilibrium when we have different kind of traders and we have and insider that is a large trader with external privilege information. We will look for the properties of the insider's optimal strategies and mainly how her behaviour affects the efficiency of the market, namely the conditions under which the market prices converge to the fundamental one. These problems have been addressed in different works, with different degrees of generality, and with very different types of insider's privileged information and demands of the uninformed traders.

In 1985 Kyle constructed the following model, he considers three kinds of actors in the market: market makers, uninformed traders and one insider who knows the fundamental or liquidation value of an asset at certain fixed released time. In the model, there is also a price function establishing the relation between the market prices and the total demand. Kyle works in the discrete time setting and with noises given by Gaussian random walks. Back (1992) extends the previous work to the continuous time case. These are seminal papers which opened the way to various generalisations and extensions. To mention some, we have Back and Pedersen (1998), who consider a dynamic fundamental price and Gaussian noises with time varying volatility; Cho (2003), who considers pricing functions depending on the path of the demand process and studies what happens when the informed trader is risk-averse; Lasserre (2004), who considers a multivariate setting; Back and Baruch (2004) where the market depth depends on the market price of the stock; Aase et al. (2012a), (2012b), who put emphasis in filtering techniques to solve the equilibrium problem; Campi and Çetin (2007), who consider a defaultable bond instead of a stock as in the Kyle-Back model and also consider the default time as privileged information; Danilova (2010), who deals with non-regular pricing rules; Caldentey and Stacchetti (2010) who take a random release time into account; Campi et al. (2013), 
who consider again a defaultable bond, but this time the privileged information is represented by some dynamic signal related with the default time; and Collin-Dufresne and Fos (2016) where the market depth depends on the (random) volatility of the noise in the market. In a previous paper by Corcuera and Di Nunno (2018), we tried to consider a general framework to include all the particular extensions mentioned above and to study the general characteristics of the equilibria. The present work intends to complete this task by including models with both, a random price pressure and a random release of information.

The paper is structured as follows. In the next section we describe the model that gives rise to the stock prices. We discuss the insider's optimal strategies for a general functional of the demand process and define the concept of admissibility for pricing rules and insider strategies. In section 3 we discuss what happens when the release time is predictable to the insider and when it is not predictable and give a characterization of the equilibrium.

\section{The model and equilibrium}

We consider a market with two assets, a stock and a bank account with interest rate $r$ equal to zero for the sake of simplicity. With abuse of terminology we will just write "prices" even though they are sometimes "discounted prices". The trading is continuous in time over the period $[0, \infty)$ and it is order driven. There is a (possibly random) release time $\tau<\infty$ a.s., when the fundamental value of the stock is revealed. The fundamental value process represents the actual value of the asset, which would be known only if all the information was public. We could say, with Malkiel (2007), that the fundamental value is the intrinsic value of the asset via an analysis of the balance sheet, the expected future dividends and the growth prospects of a company. The fundamental value process is denoted by $V$.

We shall denote the market price of the stock at time $t$ by $P_{t}$. This represents the market evaluation of the asset. Just after the revelation time the price of the stock coincides with the fundamental value. Then we consider $P_{t}$ defined only on $t \leq \tau$. Obviously, it is possible that $P_{t} \neq V_{t}$ for $t \leq \tau$.

We assume that all the random variables and processes mentioned are defined in the same complete filtered probability space $(\Omega, \mathcal{F}, \mathbb{H}, \mathbb{P})$ where the filtration $\mathbb{H}$ and any other filtration considered here are complete and right-continuous by taking, when necessary, their usual augmentation, as we shall specify below.

There are three kinds of traders. A large number of liquidity traders, who trade for liquidity or hedging reasons, an informed trader or insider, who has privileged information about the firm and can deduce its fundamental value, and the market makers, who set the market price and clear the market. 


\subsection{The agents and the equilibrium}

At time $t$, the insider information is the full information given by $\mathcal{H}_{t}$ and her flow of information is the

filtration $\mathbb{H}=\left(\mathcal{H}_{t}\right)_{t \geq 0}$. Since this is also the filtration with respect to which all the processes considered here are adapted we shall omit it in the notation. Therefore the informed trader, like any other trader, observes the market prices $P$ but, in addition, she has access to the firm value, maybe through some signal process. Moreover, she will have some knowledge about the random time $\tau$. In the sequel we will consider the two following cases:

- $\tau$ is predictable, that is there is an increasing sequence of stopping times $\left(\tau_{n}\right)$ such that a.s., $\tau_{n}<\tau$ and $\lim _{n} \tau_{n}=\tau$.

- $\tau$ is not a predictable stopping time.

We assume that the fundamental value, $V$, is a càdlàg martingale (if not otherwise specified) such that $\sigma_{V}^{2}(t):=\frac{d[V, V]_{t}^{c}}{d t}$ is well defined (where $[V, V]^{c}$ indicates the continuous part of the quadratic variation of $V$ ). Hereafter we describe in detail the three types of agents involved in this market model, namely their role, their demand process, and their information.

Let $Z$ be the aggregate demand process of the liquidity traders. We recall that these are a large number of traders motivated by liquidity or hedging reasons. They are perceived as constituting noise in the market, thus also called noise traders. It is assume that $Z$ is a continuous martingale, independent of $V$, such that $\sigma_{Z}^{2}(t):=\frac{\mathrm{d}[Z, Z]_{t}}{\mathrm{~d} t}$ is well defined. As it is shown in Corcuera et al. (2010) if $Z$ has jumps there is not equilibrium.

Remark 1 In the equilibrium model treated in this paper the random time $\tau$ and the processes $V$ and $Z$ are exogenously given.

Market makers clear the market giving the market prices. They rely on the information given by the total aggregate demand $Y$, which they observe, and the release time $\tau$, that is a stopping time for them. Hence, their information flow is: $\mathbb{F}=\left(\mathcal{F}_{t}\right)_{t \geq 0}$, where $\mathcal{F}_{t}=\bar{\sigma}\left(Y_{s}, \tau \wedge s, 0 \leq s \leq t\right)$. Here $\bar{\sigma}$ denotes the $\sigma$-field corresponding to the usual augmentation of the natural filtration (see Revuz and Yor (1999), Ch. I, Def. 4.13 and the paragraphs following this definition). That is, e.g.,

$$
\bar{\sigma}\left(Y_{s}, \tau \wedge s, 0 \leq s \leq t\right):=\bigcap_{r>t}\left(\sigma\left(Y_{s}, \tau \wedge s, 0 \leq s \leq r\right) \cup \mathcal{N}\right)
$$


Therefore $\left(P_{t}\right)_{0 \leq t \leq \tau}$ is an $\mathbb{F}$-adapted process.

The total aggregate demand is $Y:=X+Z$, where $X$ denotes the insider demand process. $X$ is naturally assumed a predictable process and it is also assumed to be a càdlàg semimartingale such that

(A1) $X_{t}=M_{t}+A_{t}+\int_{0}^{t} \theta_{s} \mathrm{~d} s$, for all $t \geq 0$, where $M$ is a continuous martingale, $M_{0}=0, A$ a bounded variation predictable process, with $A_{t}=\sum_{0<s \leq t}\left(X_{s}-X_{s-}\right)$, and $\theta$ a càdlàg adapted process.

Consistent with the original idea of Kyle (1985) and later literature, we suppose that the market makers give market prices through a pricing rule of the form:

$$
P_{t}=H\left(t, \xi_{t}\right), t \geq 0, \quad \xi_{t}:=\int_{0}^{t} \lambda\left(s, P_{s}, v_{s}\right) \mathrm{d} Y_{s}
$$

where $\lambda \in C^{1,2,2}$ is a strictly positive function that we call it price pressure, $H \in C^{1,2}, H(t, \cdot)$ is strictly increasing for every $t \geq 0$, and $v$ is an $\mathbb{F}$-adapted locally bounded process that can be seen as a market factor. We also assume, for simplicity, that

$$
\text { (H1) }[v, Y]=0 \text {. }
$$

This class of pricing rules includes that used in Kyle (1985) and Back (1992) where $\lambda$ is deterministic, in Back and Baruch (2004) where $\lambda_{s}=\lambda\left(P_{s}\right)$, and in Collin-Dufresne and Fos (2016) where $\lambda=\lambda\left(v_{s}\right)$ and $v$ is the (random) volatility of $Z$.

Now an equilibrium can be defined as

Definition 1 The triple $(H, \lambda, X)$ is an equilibrium, if the prices $P .:=H(\cdot, \xi$.$) are competitive, given X$, that is

$$
P_{t}=\mathbb{E}\left(V_{t} \mid \mathcal{F}_{t}\right), \quad 0 \leq t \leq \tau
$$

and the strategy $X$ is optimal, in the sense that it maximizes the expected final wealth of the insider, given $(H, \lambda)$, where

$$
P_{t}=H\left(t, \xi_{t}\right), \quad t \geq 0, \quad \text { with } \xi_{t}:=\int_{0}^{t} \lambda\left(s, P_{s-}, v_{s-}\right) \mathrm{d}\left(X_{s}+Z_{s}\right)
$$

Observe that since $V$ is a martingale, equality (2) implies that $P$ is an $\mathbb{F}$-martingale. Furthermore, from the 
assumptions on $Y=X+Z$ and $\lambda$, we observe that $\xi$ is a càdlàg semimartingale. Hence, applying the Itô formula to $H\left(t, \xi_{t}\right)$, we can see that $P$ is also a semimartingale.

Remark 2 From the economic point of view, due to Bertrand's type competition among market makers, in the equilibrium, market prices are rational, or competitive, in the sense that $P_{t}=\mathbb{E}\left(V_{t} \mid \mathcal{F}_{t}\right), \quad 0 \leq t \leq \tau$. The competitive price is a price such that the expectation of the market maker's profit equals zero, see Remark 4 below. Relationship (2) gives the connection between the market evaluation $P$ of the stock and its fundamental value $V$. The filtration refers to the information available to market makers.

Example 1 Assume that we look for an equilibrium, with a deterministic release time $\tau \equiv T$, in the set of insider strategies $X$ of the form

$$
X_{t}=\int_{0}^{t} \theta_{s} \mathrm{~d} s
$$

where $\theta$ is progressively adapted and that $\left(Z_{t}\right)_{t \geq 0}$, the aggregate demand of the liquidity traders, is a Brownian motion with variance $\sigma_{Z}^{2}(\cdot) \geq C>0$. Hence, the total demand is

$$
Y_{t}=\int_{0}^{t} \theta_{s} \mathrm{~d} s+\int_{0}^{t} \sigma_{Z}(s) \mathrm{d} B_{s}
$$

Then, under square integrability conditions on $\theta$ and $V$ (see Theorem 8.1 in Liptser Shiryaev (2001))

$$
\begin{aligned}
P_{t} & =\mathbb{E}\left(V_{t} \mid \mathcal{F}_{t}\right) \\
& =P_{0}+\int_{0}^{t} \frac{\mathbb{E}\left(\theta_{s} V_{s} \mid \mathcal{F}_{s}\right)-\mathbb{E}\left(V_{s} \mid \mathcal{F}_{s}\right) \mathbb{E}\left(\theta_{s} \mid \mathcal{F}_{s}\right)}{\sigma_{Z}(s)} \mathrm{d} \bar{B}_{s},
\end{aligned}
$$

where $\mathcal{F}_{t}:=\bar{\sigma}\left(Y_{s}, 0 \leq s \leq t\right)$ and

$$
\bar{B}_{t}=\int_{0}^{t} \frac{\mathrm{d} Y_{s}-\mathbb{E}\left(\theta_{s} \mid \mathcal{F}_{s}\right) \mathrm{d} s}{\sigma_{Z}(s)}
$$

is an $\mathbb{F}$-Brownian motion. Assume now that the strategy $\theta$ of the insider is of the form

$$
\theta_{s}=\beta_{s}\left(V_{s}-P_{s}\right), \quad 0 \leq s \leq T
$$

where $\left(\beta_{s}\right)_{0 \leq s \leq T}$ is deterministic. Then we have

$$
\mathbb{E}\left(\theta_{s} \mid \mathcal{F}_{s}\right)=\beta_{s}\left(\mathbb{E}\left(V_{s} \mid \mathcal{F}_{s}\right)-P_{s}\right)=0,
$$


and by the previous filtering results (4) and (5), we have that

$$
P_{t}=P_{0}+\int_{0}^{t} \lambda_{s} \mathrm{~d} Y_{s}
$$

with

$$
\lambda_{s}=\frac{\mathbb{E}\left(\theta_{s} V_{s} \mid \mathcal{F}_{s}\right)}{\sigma_{Z}^{2}(s)}=\frac{\beta_{s} \mathbb{E}\left(\left(V_{s}-P_{s}\right)^{2} \mid \mathcal{F}_{s}\right)}{\sigma_{Z}^{2}(s)}=\frac{\beta_{s} \Sigma_{s}}{\sigma_{Z}^{2}(s)}
$$

where $\Sigma_{s}:=\mathbb{E}\left(\left(V_{s}-P_{s}\right)^{2} \mid \mathcal{F}_{s}\right)$ and $Y_{t}=\int_{0}^{t} \sigma_{Z}(s) \mathrm{d} \bar{B}_{s}$. Under the additional assumption that $V_{t}=V_{0}+$ $\int_{0}^{t} \sigma_{V}(s) \mathrm{d} \tilde{B}_{s}$ where $\tilde{B}$ is another Brownian motion independent of B, then we have (see Theorem 12.1. Liptser Shiryaev (2001))

$$
\frac{\mathrm{d}}{\mathrm{d} t} \Sigma_{t}=\sigma_{V}^{2}(t)-\frac{\beta_{t}^{2} \Sigma_{s}^{2}}{\sigma_{Z}^{2}(t)}=\sigma_{V}^{2}(t)-\lambda_{t}^{2} \sigma_{Z}^{2}(t)
$$

So given that prices are of the form $P_{t}=P_{0}+\int_{0}^{t} \lambda_{s} \mathrm{~d} Y_{s}$ (the only competitive prices for the strategies $\left.\theta_{s}=\beta_{s}\left(V_{s}-P_{s}\right)\right)$, if we have an equilibrium we need that

$$
\beta_{t}=\frac{\lambda_{t} \sigma_{Z}^{2}(t)}{\Sigma_{0}+\int_{0}^{t} \sigma_{V}^{2}(s) \mathrm{d} s-\int_{0}^{t} \lambda_{s}^{2} \sigma_{Z}^{2}(s) \mathrm{d} s}
$$

But, how do we obtain $\lambda_{t}$ ? What about the optimality of $\beta_{t}$ ?

Remark 3 In this simple example we see that if we confine the set of admissible strategies of the insider sufficiently, the way in which prices depend on the total aggregate demand in an equilibrium is almost determined (up to a parameter!) by the rationality of prices. Later we shall call this fact dependency on a pricing rule.

The informed trader is assumed risk-neutral and she aims at maximizing her expected final wealth. To obtain the formula for the insider's wealth assume that trades are made at times $0 \leq t_{1} \leq t_{2} \leq \ldots \leq t_{N}=\tau$. If at time $t_{i-1}$ there is an order to buy $X_{t_{i}}-X_{t_{i-1}}$ shares, its cost will be $P_{t_{i}}\left(X_{t_{i}}-X_{t_{i-1}}\right)$, so there is a change in the bank account given by

$$
-P_{t_{i}}\left(X_{t_{i}}-X_{t_{i-1}}\right)
$$

and due to the fact that at the release time $\tau$ the price of the asset becomes the fundamental one, there is the extra income: $X_{\tau} V_{\tau}$ then we have

$$
\begin{aligned}
W_{\tau} & =-\sum_{i=1}^{N} P_{t_{i}}\left(X_{t_{i}}-X_{t_{i-1}}\right)+X_{\tau} V_{\tau} \\
& =-\sum_{i=1}^{N} P_{t_{i-1}}\left(X_{t_{i}}-X_{t_{i-1}}\right)-\sum_{i=1}^{N}\left(P_{t_{i}}-P_{t_{i-1}}\right)\left(X_{t_{i}}-X_{t_{i-1}}\right)+X_{\tau} V_{\tau},
\end{aligned}
$$


so, if the time between trades goes to zero, we will have

$$
W_{\tau}=-\int_{0}^{\tau} P_{t-} \mathrm{d} X_{t}-[P, X]_{\tau}+X_{\tau} V_{\tau}
$$

Alternatively, by integrating by parts we can write,

$$
W_{\tau}=\int_{0}^{\tau}\left(V_{t-}-P_{t-}\right) \mathrm{d} X_{t}+[V, X]_{\tau}-[P, X]_{\tau}+\int_{0}^{\tau} X_{t-} \mathrm{d} V_{t}
$$

Remark 4 Note that, by construction, $X$ is predictable since $X_{t_{i}}-X_{t_{i-1}}$ is the number of assets ordered at time $t_{i-1}$. On the other hand these assets are bought at price $P_{t_{i}}$, the market price at time $t_{i}$ (that is affected by the total demand of the asset). So we are in the context of an auction mechanism. If and order emitted at time $t_{i-1}$ was executed at prices at time $t_{i-1}$ we would have the alternative expression for the final wealth, assuming that $X_{0}=0$, given by

$$
\begin{aligned}
W_{\tau} & =-\sum_{i=1}^{N} P_{t_{i-1}}\left(X_{t_{i}}-X_{t_{i-1}}\right)+X_{\tau} P_{\tau}+X_{\tau}\left(V_{\tau}-P_{\tau}\right) \\
& =-\sum_{i=1}^{N} P_{t_{i-1}}\left(X_{t_{i}}-X_{t_{i-1}}\right)+\sum_{i=1}^{N}\left(X_{t_{i}} P_{t_{i}}-X_{t_{i-1}} P_{t_{i-1}}\right)+X_{\tau}\left(V_{\tau}-P_{\tau}\right) \\
& =\sum_{i=1}^{N} X_{t_{i}}\left(P_{t_{i}}-P_{t_{i-1}}\right)+X_{\tau}\left(V_{\tau}-P_{\tau}\right)
\end{aligned}
$$

that corresponds to a self-financed portfolio with initial value equal to zero and an additional injection of money at time $\tau$ given by

$$
X_{\tau}\left(V_{\tau}-P_{\tau}\right)
$$

This happens for the market makers investment. Their total wealth is given by

$$
W_{\tau}^{M}:=\sum_{i=1}^{N} Y_{t_{i}}\left(P_{t_{i}}-P_{t_{i-1}}\right)+X_{\tau}\left(V_{\tau}-P_{\tau}\right)
$$

and since prices are competitive $\mathbb{E}\left(W_{\tau}^{M}\right)=0$.

For notational reasons we give the following definition:

Definition 2 Let $S$ be a semimartingale with canonical decomposition $S=N+A$, where $N$ is a squareintegrable martingale and $A$ is a predictable finite-variation process. Let $\nu$ be a stopping time and $U$ be an 
adapted process, we write $U \in L_{\nu}(S)$ to indicate that

$$
\mathbb{E}\left(\int_{0}^{\nu} U_{t-}^{2} \mathrm{~d}[N, N]_{t}+\int_{0}^{\nu}\left|U_{t-}\right|\left|\mathrm{d} A_{t}\right|\right)<\infty
$$

In the next subsection we discuss the characterization of an insider's optimal strategy in equilibrium in terms of fundamental value and insider information. In the sequel we will consider two kinds of stopping times: $\tau$ bounded, or $\tau$ finite but independent of the rest of observable random variables. In both cases, by the assumptions that $V$ is a martingale and $X$ a predictable càdlàg semimartingale satisfying that $X \in L_{\tau}(V)$ (see below), we have that $\mathbb{E}\left(\int_{0}^{\tau} X_{t} \mathrm{~d} V_{t}\right)=0$. In fact, we can argue that, if $\tau$ is bounded, we can apply Doob's Optional Sampling Theorem and, if $\tau$ is finite but independent of $(V, P, Z, \ldots)$ (and consequently of $X$ ), we have that

$$
\mathbb{E}\left(\int_{0}^{\tau} X_{t-} \mathrm{d} V_{t}\right)=\mathbb{E}\left(\mathbb{E}\left(\int_{0}^{\tau} X_{s-} \mathrm{d} V_{s} \mid \tau\right)\right)=\mathbb{E}\left(\left.\mathbb{E}\left(\int_{0}^{t} X_{s-} \mathrm{d} V_{s}\right)\right|_{t=\tau}\right)=0
$$

Hence,

$$
J(X):=\mathbb{E}\left(W_{\tau}\right)=\mathbb{E}\left(\int_{0}^{\tau}\left(V_{t-}-P_{t-}\right) \mathrm{d} X_{t}+[V, X]_{\tau}-[P, X]_{\tau}\right)
$$

First, we have the following proposition that simplifies the set of admissible strategies to take into account.

Proposition 1 Strategies with a continuous martingale part or with jumps are suboptimal, in the sense that they do not maximize the expected value of (6), in the class of all predictable semimartingale strategies.

Proof. Since we assume that prices increase with $Y=X+Z$, strategies with a continuous martingale part or predictable jumps will be suboptimal in the class of semimartingale strategies: in fact in (6) we can approximate these strategies by a continuous process with zero quadratic variation and then eliminating the negative contribution of $[P, X]_{\tau}$ and at the same time approximating, as much as we want, the rest of the terms in (6).

rule0.5em0.5em

As a consequence, to find an equilibrium, we can change (A1) above by

$$
\left(\mathbf{A 1}^{\prime}\right) X_{t}=\int_{0}^{t} \theta_{s} \mathrm{~d} s, \text { for all } t \geq 0, \text { where } \theta \text { a càdlàg adapted process. }
$$

and the goal of the insider becomes to maximize $J(X)$ with

$$
J(X):=\mathbb{E}\left(W_{\tau}\right)=\mathbb{E}\left(\int_{0}^{\tau}\left(V_{t}-P_{t}\right) \mathrm{d} X_{t}\right) .
$$


We also have a general result in the case that $\tau$ is a predictable time to the insider.

Proposition 2 If $\tau$ is a predictable stopping time to the insider and $X$ is an optimal strategy, then we have market efficiency

$$
V_{\tau-}=P_{\tau-}=P_{\tau} \text { a.s. }
$$

Proof. If the insider's strategy is such that $V_{\tau-}-P_{\tau-} \neq 0$ then it is suboptimal since the insider could approximate a jump at $\tau$ with the same sign to $V_{\tau-}-P_{\tau-}$ by an absolutely continuous strategy and improving her wealth. Now, since $V$ is a martingale and $\tau$ is predictable $\mathbb{E}\left(V_{\tau} \mid \mathcal{F}_{\tau-}\right)=V_{\tau-}$ (see Jacod and Shiryaev (1987), Lemma 2.27). (We recall that $\mathcal{F}_{\tau-}:=\mathcal{F}_{0} \vee \sigma\left(A \cap(\tau>t): A \in \mathcal{F}_{t}, t \geq 0\right)$, see, e.g., Revuz and Yor (1999), page 46). Then there is not extra income, in average, by the possible jump of $V$ at $\tau$. rule0.5em0.5em

Remark 5 That the market efficiency is a consequence of the optimality of the insider's strategy was already observed, for instance, in Aase et al. (2012a). Here we obtain an extension of this result for a more general behaviour of the fundamental value, the demand process of the noise traders and the pricing rules.

Remark 6 This efficiency situation is also the case in Campi and Çetin (2007). Here the insider knows the default time, $\bar{\tau}$, of a bond with face value 1 and consequently its fundamental value $V_{t}=\mathbf{1}_{\{\bar{\tau}>1\}}$, and the release time is $\tau=\bar{\tau} \wedge 1$. So, $\tau$ is $\mathcal{F}_{0}$-measurable and bounded. Then, they obtain

$$
\mathbf{1}_{\{\bar{\tau}>1\}}-P_{\bar{\tau} \wedge 1}=0 \quad \text { a.s. }
$$

Within this study, the authors also assume that $\bar{\tau}$ is the first passage time of a standard Brownian motion independent of demand of the noise traders $Z$.

Remark 7 If we take the fundamental value $V_{t} \equiv V$ and the deterministic fixed release time $\tau \equiv 1$, then we retrieve Back's framework (1992). There it is shown that market prices converge to $V$ when $t \rightarrow 1$.

In order to find the first order conditions of the optimal strategy we study the effect of an $\varepsilon$-perturbation of an optimal strategy: $\mathrm{d} X_{t}^{(\varepsilon)}:=\mathrm{d} X_{t}+\varepsilon \beta_{t} \mathrm{~d} t$, where $\beta$ is a bounded adapted processes. We denote

$$
P_{t}^{(\varepsilon)}=H\left(t, \xi_{t}^{(\varepsilon)}\right), \quad \xi_{t}^{(\varepsilon)}=\int_{0}^{t} \lambda\left(s, P_{s}^{(\varepsilon)}, v_{s}\right) \mathrm{d}\left(X_{s}^{(\varepsilon)}+Z_{s}\right)
$$


And we assume that pricing rules (3) are Gateaux differentiable in such a way that

$$
\text { (R) } P_{t}^{(\varepsilon)}-P_{t}=\varepsilon \int_{0}^{t} K(s, t) \beta_{s} \mathrm{~d} s+o(\varepsilon) R_{t}
$$

for a family of strictly positive random variables $K(s, t)(\omega), \mathcal{B}\left(\mathbb{R}_{+}\right) \otimes \mathcal{P}^{\mathbb{F}}$ - measurable and for all $0 \leq s \leq t \leq \tau$ and $R$ a bounded progressively measurable process.

From now on, we shall write $\partial_{i}, \partial_{i j}$ to denote the first and second derivatives with respect to the $i^{t h}, i^{t h}$ and $j^{j h}$ variables, respectively. We have the following proposition.

Proposition 3 Assume that the market factor $v$ in the price pressure $\lambda$ (see (1)) is not affected by the strategy of the insider. For any bounded adapted process $\beta$, if we take $\mathrm{d} X_{t}^{(\varepsilon)}=\mathrm{d} X_{t}+\varepsilon \beta_{t} \mathrm{~d} t$, then we have

$$
\left.\frac{\mathrm{d} P_{t}^{(\varepsilon)}}{\mathrm{d} \varepsilon}\right|_{\varepsilon=0}=\partial_{2} H\left(t, \xi_{t}\right) \eta_{t} \int_{0}^{t} \frac{\lambda\left(s, P_{s}, v_{s}\right)}{\eta_{s}} \beta_{s} \mathrm{~d} s
$$

with

$$
\eta_{t}:=\mathcal{E}\left(\int_{0}^{t} \partial_{2} H \partial_{2} \lambda \mathrm{d} Y_{s}\right)
$$

where $\mathcal{E}$ denotes the stochastic exponential.

Proof.

$$
\left.\frac{\mathrm{d} P_{t}^{(\varepsilon)}}{\mathrm{d} \varepsilon}\right|_{\varepsilon=0}=\partial_{2} H\left(\left.\int_{0}^{t} \partial_{2} \lambda\left(s, P_{s}, v_{s}\right) \frac{\mathrm{d} P_{s}^{(\varepsilon)}}{\mathrm{d} \varepsilon}\right|_{\varepsilon=0} \mathrm{~d} Y_{s}+\int_{0}^{t} \lambda\left(s, P_{s}, v_{s} \theta_{s}\right) \beta_{s} \mathrm{~d} s\right),
$$

so

$$
\frac{\left.\frac{\mathrm{d} P_{t}^{(\varepsilon)}}{\mathrm{d} \varepsilon}\right|_{\varepsilon=0}}{\partial_{2} H}=\eta_{t} \int_{0}^{t} \frac{\lambda\left(s, P_{s}, v_{s}\right)}{\eta_{s}} \beta_{s} \mathrm{~d} s
$$

with

$$
\eta_{t}:=\mathcal{E}\left(\int_{0}^{t} \partial_{2} H \partial_{2} \lambda \mathrm{d} Y_{s}\right)
$$


In fact

$$
\begin{aligned}
\mathrm{d}\left(\eta_{t} \int_{0}^{t} \frac{\lambda\left(s, P_{s}, v_{s}\right)}{\eta_{s}} \beta_{s} \mathrm{~d} s\right) & =\mathrm{d} \eta_{t} \int_{0}^{t} \frac{\lambda\left(s, P_{s}, v_{s}\right)}{\eta_{s}} \beta_{s} \mathrm{~d} s+\eta_{t} \frac{\lambda\left(t, P_{t}, v_{t}\right)}{\eta_{t}} \beta_{t} \mathrm{~d} t \\
& =\eta_{t} \partial_{2} H \partial_{2} \lambda \mathrm{d} Y_{t} \int_{0}^{t} \frac{\lambda\left(s, P_{s}, v_{s}\right)}{\eta_{s}} \beta_{s} \mathrm{~d} s+\lambda\left(t, P_{t}, v_{t}\right) \beta_{t} \mathrm{~d} t \\
& =\left.\frac{\mathrm{d} P_{t}^{(\varepsilon)}}{\mathrm{d} \varepsilon}\right|_{\varepsilon=0} \partial_{2} \lambda \mathrm{d} Y_{t}+\lambda\left(t, P_{t}, v_{t}\right) \beta_{t} \mathrm{~d} t \\
& =\mathrm{d}\left(\frac{\left.\frac{\mathrm{d} P_{t}^{(\varepsilon)}}{\mathrm{d} \varepsilon}\right|_{\varepsilon=0}}{\partial_{2} H}\right)
\end{aligned}
$$

This completes the proof.

rule0.5em0.5em

Therefore in $(\mathbf{R})$ we have

$$
K(s, t)=\frac{\lambda\left(s, P_{s}, v_{s}\right)}{\eta_{s}} \partial_{2} H\left(t, \xi_{t}\right) \eta_{t}
$$

with the factorization $K(s, t)=K_{1}(s) K_{2}(t)$ where

$$
K_{1}(s)=\frac{\lambda\left(s, P_{s}, v_{s}\right)}{\eta_{s}}, K_{2}(t)=\partial_{2} H\left(t, \xi_{t}\right) \eta_{t}
$$

Remark 8 In Laserre (2004) the author considers pricing rules, in a multivariate setting, of the form

$$
P_{t}^{i}=H^{i}\left(t, \xi_{t}^{1}, \ldots, \xi_{t}^{n}\right), t \geq 0, \quad \xi_{t}^{i}:=\int_{0}^{t} \lambda^{i}(s) \mathrm{d} Y_{s}^{i}, \quad i=1, \ldots, n,
$$

In such a way that

$$
K^{i}(s, t)=\lambda^{i}(s) \partial_{i} H^{i}\left(t, \xi_{t}^{1}, \ldots, \xi_{t}^{n}\right)
$$

where $\partial_{i} H, \partial_{i j} H$ denote the first and second derivatives with respect to the $i^{\text {th }}$, $i^{\text {th }}$ and $j^{j h}$ variables, respectively. So we can extend the univariate results to this setting in a straightforward manner.

\section{The optimality condition}

For technical and modelling reasons, we require additional properties to $P$ and the strategy $X$. 
Definition 3 We say that the triple $(H, \lambda, X)$ is admissible if it satisfies $(1),(\mathbf{H 1}),\left(\mathbf{A} \mathbf{1}^{\prime}\right),(\mathbf{R})$ and also

(A2) $X \in L_{\tau}(V), V, P \in L_{\tau}(X) \cap L_{\tau}(Z)$,

(АЗ) $K_{2}(\cdot)\left(\int_{0}^{\cdot} K_{1}(s)^{2} \mathrm{~d} s\right)^{1 / 2} \in L_{\tau}(X)$

We have the following theorem.

Theorem 1 If $(H, \lambda, X)$ is admissible and $X$ is optimal we have

$$
V_{t}-H\left(t, \xi_{t}\right)-\frac{\lambda_{t}}{\eta_{t}} \mathbb{E}\left(\int_{t}^{\tau} \partial_{2} H\left(s, \xi_{s}\right) \eta_{s} \mathrm{~d} X_{s} \mid \mathcal{F}_{t}\right)=0, \text { a.s. }-\mathbb{P} \otimes \text { Leb on }[[0, \tau)) .
$$

where

$$
\eta_{t}:=\mathcal{E}\left(\int_{0}^{t} \partial_{2} H \partial_{2} \lambda \mathrm{d} Y_{s}\right), 0 \leq t<\tau
$$

Proof. Take $\mathrm{d} X_{t}^{(\varepsilon)}:=\mathrm{d} X_{t}+\varepsilon \beta_{t} \mathrm{~d} t$, where $\beta$ is a bounded adapted processes, then taking the admissibility conditions in Definition 3 into account, we have

$$
\begin{aligned}
& \mathbb{E}\left(W_{\tau}^{(\varepsilon)}-W_{\tau}\right) \\
& \left.=\mathbb{E}\left(\int_{0}^{\tau}\left(V_{t}-P_{t}^{(\varepsilon)}\right) \mathrm{d} X_{t}^{(\varepsilon)}-W_{\tau}\right)\right) \\
& =\varepsilon \mathbb{E}\left(\left(\int_{0}^{\tau}\left(V_{t}-P_{t}\right) \beta_{t} \mathrm{~d} t-\int_{0}^{\tau}\left(\int_{0}^{t} K(s, t) \beta_{s} \mathrm{~d} s\right) \mathrm{d} X_{t}\right)\right)+o(\varepsilon) \\
& =\varepsilon \mathbb{E}\left(\left(\int_{0}^{\tau}\left(V_{t}-P_{t}-\int_{t}^{\tau} K(t, s) \mathrm{d} X_{s}\right) \beta_{t} \mathrm{~d} t\right)\right)+o(\varepsilon) .
\end{aligned}
$$

Note that condition (A3) guarantees that

$$
\mathbb{E}\left(\int_{0}^{\tau}\left(\int_{0}^{t} K(s, t) \beta_{s} \mathrm{~d} s\right) \mathrm{d} X_{t}\right)=\mathbb{E}\left(\int_{0}^{\tau}\left(\int_{t}^{\tau} K(t, s) \mathrm{d} X_{s}\right) \beta_{t} \mathrm{~d} t\right)
$$

Then

$$
\left.\frac{\mathrm{d} \mathbb{E}\left(W_{\tau}^{(\varepsilon)}\right)}{\mathrm{d} \varepsilon}\right|_{\varepsilon=0}=0
$$

implies, by (R), that

$$
\mathbb{E}\left(\int_{0}^{\tau}\left(V_{t}-P_{t}-\int_{t}^{\tau} K(t, s) \mathrm{d} X_{s}\right) \beta_{t} \mathrm{~d} t\right)=0
$$

and since we can take $\beta_{t}=\alpha_{u} \mathbf{1}_{(u, u+h]}(t)$, with $\alpha_{u}$ measurable and bounded and $\tau$ is a stopping time, we 
have that

$$
V_{t}-P_{t}-\mathbb{E}\left(\int_{t}^{\tau} K(t, s) \mathrm{d} X_{s} \mid \mathcal{H}_{t}\right)=0
$$

a.s. $\mathbb{P} \otimes L e b$ on the stochastic interval $[[0, \tau))$. Finally, since

$$
K(t, s)=\frac{\lambda\left(t, P_{t}, v_{t}\right)}{\eta_{t}} \partial_{2} H\left(s, \xi_{s}\right) \eta_{s}
$$

we obtain the result.

rule0.5em0.5em

\subsection{Case when $\tau$ is predictable to the insider}

In this section we consider the case when the insider can predict the release time of information $\tau$. Namely, there is an increasing sequence of stopping times $\left(\tau_{n}\right)$ such that a.s., $\tau_{n}<\tau$ and $\lim _{n} \tau_{n}=\tau$. Moreover, we assume that $\tau$ is bounded.

\subsubsection{Necessary conditions for the equilibrium}

We have the following Lemma.

Lemma 1 Under the pricing rules considered above, we have

$$
\left[P, \frac{\eta}{\lambda}\right] \equiv 0
$$

Proof. By using the Itô formula together with the condition (H1) we obtain that

$$
\begin{aligned}
\mathrm{d}\left(\frac{\eta_{s}}{\lambda_{s}}\right) & =\eta_{s} \mathrm{~d}_{v}\left(\frac{1}{\lambda_{s}}\right)+\frac{1}{\lambda_{s}} \mathrm{~d} \eta_{s}+\mathrm{d}\left[\eta, \frac{1}{\lambda}\right]_{s} \\
& =\eta_{s} \mathrm{~d}_{v}\left(\frac{1}{\lambda}\right)-\eta_{s} \frac{\partial_{y} \lambda_{s}}{\lambda_{s}^{2}} \lambda_{s} \mathrm{~d} Y_{s}-\frac{1}{2} \eta_{s} \frac{\lambda_{s}^{2} \partial_{y y} \lambda_{s}-2\left(\partial_{y} \lambda_{s}\right)^{2} \lambda_{s}}{\lambda_{s}^{4}} \lambda_{s}^{2} \sigma_{Y}^{2} \mathrm{~d} s \\
& -\eta_{s} \frac{\partial_{s} \lambda_{s}}{\lambda_{s}^{2}} \mathrm{~d} s+\frac{1}{\lambda_{s}} \eta_{s} \partial_{y} \lambda_{s} \mathrm{~d} Y_{s}-\frac{\left(\partial_{y} \lambda_{s}\right)^{2}}{\lambda_{s}} \sigma_{Y}^{2} \eta_{s} \mathrm{~d} s \\
& =\eta_{s} \mathrm{~d}_{v}\left(\frac{1}{\lambda}\right)-\frac{1}{2} \frac{\lambda_{s} \partial_{y y} \lambda-2\left(\partial_{y} \lambda_{s}\right)^{2}}{\lambda_{s}} \sigma_{Y}^{2} \eta_{s} \mathrm{~d} s-\frac{\left(\partial_{y} \lambda_{s}\right)^{2}}{\lambda_{s}} \sigma_{Y}^{2} \mathrm{~d} s \\
& =\eta_{s} \mathrm{~d}_{v}\left(\frac{1}{\lambda}\right)-\eta_{s}\left(\frac{1}{2} \partial_{y y} \lambda_{s} \sigma_{Y}^{2}+\frac{\partial_{1} \lambda_{s}}{\lambda_{s}^{2}}\right) \mathrm{d} s,
\end{aligned}
$$

where $\partial_{y} \lambda_{s}=\partial_{2} \lambda_{s} \partial_{2} H_{s}$ and $\mathrm{d}_{v}$ the differential w.r.t. $v$. Again by condition $(\mathbf{H} 1)$ and $(3)\left[P, \int_{0}^{\cdot} \mathrm{d}_{v}\left(\frac{1}{\lambda}\right)\right]=0$, 
and this together with (11) gives (10).

rule0.5em0.5em

Furthermore, we obtain the following result, where we omit the arguments in $H$ and $\lambda$.

Proposition 4 If $(H, \lambda, X)$ is an equilibrium, we have

$$
\begin{aligned}
& \text { (i) } \frac{\mathrm{d}\left(\frac{\eta}{\lambda}\right)_{t}^{a c}}{\mathrm{~d} t}\left(V_{t}-H_{t}\right)-\left(\frac{\eta}{\lambda}\right)_{t}\left(\partial_{1} H_{t}+\frac{1}{2} \partial_{22} H_{t} \lambda_{t}^{2} \sigma_{Z}^{2}(t)\right)=0, \\
& \text { (ii) } \partial_{1} H_{t}+\frac{1}{2} \partial_{22} H_{t} \lambda_{t}^{2} \sigma_{Z}^{2}(t)=0
\end{aligned}
$$

a.s. on $[[0, \tau))$. Here $\left(\frac{\eta}{\lambda}\right)^{a c}$ indicates the absolutely continuous part of the process $\frac{\eta}{\lambda}$.

Proof. (i) By Itô's formula, we have that

$$
\begin{aligned}
\int_{t}^{\tau} \partial_{2} H\left(s, \xi_{s}\right) \eta_{s} \mathrm{~d}\left(X_{s}+Z_{s}\right) & =\int_{t}^{\tau} \frac{\eta_{s}}{\lambda_{s}} \partial_{2} H\left(s, \xi_{s}\right) \mathrm{d} \xi_{s}=\int_{t}^{\tau} \frac{\eta_{s}}{\lambda_{s}}\left(\mathrm{~d} P_{s}-\partial_{1} H_{t}-\frac{1}{2} \partial_{22} H_{t} \lambda_{t}^{2} \sigma_{Z}^{2}(t) \mathrm{d} s\right) \\
& =\frac{\eta_{\tau}}{\lambda_{\tau}} P_{\tau}-\frac{\eta_{t}}{\lambda_{t}} P_{t}-\int_{t}^{\tau} P_{s} \mathrm{~d}\left(\frac{\eta_{s}}{\lambda_{s}}\right)-\left[P, \frac{\eta}{\lambda}\right]_{t}^{\tau}-\int_{t}^{\tau} \frac{\eta_{s}}{\lambda_{s}}\left(\partial_{1} H_{t}+\frac{1}{2} \partial_{22} H_{t} \lambda_{t}^{2} \sigma_{Z}^{2}(t)\right) \mathrm{d} s
\end{aligned}
$$

Now, by (10), Equation (9) and (12), for all $t \geq 0$

$$
\begin{aligned}
0 & =\mathbf{1}_{[0, \tau)}(t)\left(V_{t}-P_{t}\right) \frac{\eta_{t}}{\lambda_{t}}+\frac{\eta_{t \wedge \tau}}{\lambda_{t \wedge \tau}} P_{t \wedge \tau}-\int_{0}^{t \wedge \tau} P_{s} \mathrm{~d}\left(\frac{\eta_{s}}{\lambda_{s}}\right)-\int_{0}^{t \wedge \tau} \frac{\eta_{s}}{\lambda_{s}}\left(\partial_{1} H_{t}+\frac{1}{2} \partial_{22} H_{t} \lambda_{t}^{2} \sigma_{Z}^{2}(t)\right) \mathrm{d} s \\
& +\mathcal{M}_{t}
\end{aligned}
$$

where $\mathcal{M}$ is a Lévy $\mathbb{H}$-martingale. Observe that the jump at $\tau$ of $\mathbf{1}_{[0, \tau)}(t)$ is killed by the fact that $V_{\tau-}-P_{\tau-}=$ 0 , and recall the uniqueness of the canonical decomposition of (special) semimartingales. Then, we obtain (i). Recall that $(H, \lambda, X)$ is an equilibrium and that the prices are rational given $X$. So, by taking conditional expectations with respect to $\mathcal{F}_{t}$ in $(i)$ we have $(i i)$.

rule0.5em0.5em

As a consequence we have the following corollaries.

Corollary 1 Assume that $(H, \lambda, X)$ is an equilibrium. In the case when $\lambda$ is independent of $v$ and $V_{t} \neq P_{t}$ a.s. (except for a set with $\mathbb{P} \otimes$ Leb zero measure) on $[[0, \tau))$ we have that

$$
\partial_{t} \lambda\left(t, P_{t}\right)+\frac{1}{2} \lambda^{2}\left(t, P_{t}\right) \partial_{y y} \lambda\left(t, P_{t}\right) \sigma_{Y}^{2}(t)=0 \text { a.s. on }[[0, \tau)) \text {. }
$$


Proof. From $(i)$ in the previous proposition we have that

$$
\frac{\mathrm{d}\left(\frac{\eta}{\lambda}\right)_{t}^{a c}}{\mathrm{~d} t}\left(V_{t}-H\left(t, \xi_{t}\right)\right)=0
$$

then $V_{t} \neq H\left(t, \xi_{t}\right)$ implies that

$$
\frac{\mathrm{d}\left(\frac{\eta}{\lambda}\right)_{t}^{a c}}{\mathrm{~d} t}=0
$$

Now, since $\lambda$ is independent of $v$, from (11) we conclude that

$$
\frac{1}{2} \partial_{y y} \lambda_{s} \sigma_{Y}^{2}+\frac{\partial_{t} \lambda_{s}}{\lambda_{t}^{2}}=0
$$

and we obtain (14).

rule0.5em0.5em

Corollary 2 Assume that $(H, \lambda, X)$ is an equilibrium, then we have:

(i) $Y$ is an $\mathbb{F}$-local martingale;

(ii) If $V_{t} \neq P_{t}$ a.s. (except for a set with $\mathbb{P} \otimes$ Leb zero measure) on $[[0, \tau))$, then $\frac{\eta}{\lambda}$ is a positive local martingale.

Proof. (i) From (ii) in Proposition 4, we have that

$$
\partial_{t} H\left(t, \xi_{t}\right)+\frac{1}{2} \partial_{y y} H\left(t, \xi_{t}\right) \lambda_{t}^{2} \sigma_{Z}^{2}(t)=0
$$

and consequently

$$
\mathrm{d} P_{t}=\mathrm{d} H\left(t, \xi_{t}\right)=\lambda_{t} \partial_{y} H\left(t, \xi_{t}\right) \mathrm{d} Y_{t}
$$

Now, since $P$ is an $\mathbb{F}$-martingale in an equilibrium and $\lambda_{t} \partial_{y} H(t, y)>0$, we have that $Y$ is an $\mathbb{F}$ - local martingale.

(ii) From (i) in Proposition 4 and (15) we have that

$$
\frac{\mathrm{d}\left(\frac{\eta}{\lambda}\right)_{t}^{a c}}{\mathrm{~d} t}\left(V_{t}-H\left(t, \xi_{t}\right)\right)=0
$$

then $V_{t} \neq H\left(t, \xi_{t}\right)$ implies that

$$
\frac{\mathrm{d}\left(\frac{\eta}{\lambda}\right)_{t}^{a c}}{\mathrm{~d} t}=0
$$

and this concludes the proof. 
rule0.5em0.5em

Example 2 As a continuation of Example 1, by the previous results about the necessary conditions for an equilibrium, if $\lambda_{t}=\lambda(t)$ then $\eta_{t} \equiv 1$ and $\lambda$ has to be a constant. Moreover the efficiency condition implies that $\Sigma_{T}=0$. These two conditions allow us to find the equilibrium in this example:

$$
\frac{\mathrm{d}}{\mathrm{d} t} \Sigma_{t}=\sigma_{V}^{2}(t)-\frac{\beta_{t}^{2} \Sigma_{s}^{2}}{\sigma_{Z}^{2}(t)}=\sigma_{V}^{2}(t)-\lambda_{t}^{2} \sigma_{Z}^{2}(t)
$$

so,

$$
\lambda=\sqrt{\frac{\Sigma_{0}+\int_{0}^{T} \sigma_{V}^{2}(t) \mathrm{d} t}{\int_{0}^{T} \sigma_{Z}^{2}(t) \mathrm{d} t}}
$$

since $\Sigma_{T}=0$, and finally

$$
\beta_{t}=\frac{\lambda \sigma_{Z}^{2}(t)}{\lambda^{2} \int_{t}^{T} \sigma_{Z}^{2}(s) \mathrm{d} s-\int_{t}^{T} \sigma_{V}^{2}(s) \mathrm{d} s} .
$$

This is the only possible equilibrium but we should check if the necessary conditions to have a maximum are really sufficient. We will discuss it in the next subsection.

Example 3 In our model consider the case when $H(t, x)=x ; \lambda=\lambda(t, x), \mathrm{d} Z_{t}=\sigma_{t} \mathrm{~d} B_{t}$, where $B$ is a Brownian motion, and when the class of admissible strategies are absolutely continuous: $\mathrm{d} X_{t}=\theta_{t} \mathrm{~d} t$. Then, we have that

$$
\mathrm{d}\left(\frac{\eta_{s}}{\lambda_{s}}\right)=-\eta_{s}\left(\frac{1}{2} \partial_{x x} \lambda_{s} \sigma_{s}^{2}+\frac{\partial_{s} \lambda_{s}}{\lambda_{s}^{2}}\right) \mathrm{d} s
$$

Then if $V_{t}-P_{t} \neq 0$ this implies that $\partial_{s} \lambda_{s}+\frac{1}{2} \partial_{x x} \lambda_{s} \lambda_{s}^{2} \sigma_{s}^{2}=0$, so $\lambda_{t}=\lambda\left(t, P_{t}\right)$ is also a local martingale. In particular if $\lambda_{t}=\lambda\left(P_{t}\right)$, it is easy to see that market prices follow a Black-Scholes model or a Bachelier model. In fact we have that

$$
\partial_{x x} \lambda_{s}=0
$$

and that $\frac{\eta}{\lambda}$ is a constant. Notice that, since prices are $\mathbb{F}$-martingales, $\lambda$ is also an $\mathbb{F}$-martingale, as it is a linear function of them. Also we have

$$
\begin{aligned}
\lambda_{t} & =a \eta_{t} \\
& =a \exp \left(\int_{0}^{t} \partial_{x} \lambda\left(P_{s}\right) \mathrm{d} Y_{s}-\frac{1}{2} \int_{0}^{t}\left(\partial_{x} \lambda\left(P_{s}\right)\right)^{2} \sigma_{s}^{2} \mathrm{~d} s\right) \\
& =a \exp \left(c Y_{t}-\frac{c^{2}}{2} \int_{0}^{t} \sigma_{s}^{2} \mathrm{~d} s\right) \\
& =c P_{t}+b
\end{aligned}
$$


and, if $c \neq 0$,

$$
P_{t}-P_{0}=a \int_{0}^{t} \exp \left(c Y_{s}-\frac{c^{2}}{2} \int_{0}^{s} \sigma_{u}^{2} \mathrm{~d} u\right) \mathrm{d} Y_{s}=\frac{a}{c} \exp \left(c Y_{t}-\frac{c^{2}}{2} \int_{0}^{t} \sigma_{s}^{2} \mathrm{~d} s\right)-\frac{a}{c}
$$

Then taking $P_{0}=\frac{a}{c}$

$$
P_{t}=P_{0} \exp \left(c Y_{t}-\frac{c^{2}}{2} \int_{0}^{t} \sigma_{s}^{2} \mathrm{~d} s\right)
$$

and $b=0$ (Black-Scholes model). If $c=0$ then $\lambda_{t}$ is constant and $P_{t}=P_{0}+b Y_{t}$ (Bachelier model).

Example 4 In Collin-Dufresne and Fos (2016), the authors take strategies $X$ absolutely continuous of the form

$$
\mathrm{d} X_{t}=\beta_{t}\left(V-P_{t}\right) \mathrm{d} t
$$

and assume that $\mathrm{d} Z_{t}=\sigma_{z}(t) \mathrm{d} B_{t}^{z}$, where $\sigma_{z}(t)$ is a semimartingale independent of the other processes, $\beta_{t}$ can depend on $\sigma_{z}(t)$ and $V$ is Gaussian. Notice that $\sigma_{z}$ is $\mathbb{F}$-adapted since corresponds to the volatility of $Y$. Now, by filtering results, we have

$$
\lambda_{t}=\frac{\Sigma_{t} \beta_{t}}{\sigma_{z}^{2}(t)}
$$

and

$$
\frac{\mathrm{d}}{\mathrm{d} t} \Sigma_{t}=-\frac{\left(\Sigma_{t} \beta_{t}\right)^{2}}{\sigma_{z}^{2}(t)}=-\lambda_{t}^{2} \sigma_{z}^{2}(t)
$$

We look for a solution of the form,

$$
\lambda_{t}=\sqrt{\frac{\Sigma_{t}}{G_{t}}}
$$

where, according to (ii) in Corollary 2, $\left(G_{t}\right)$ has to be a process such that $\left(\frac{1}{\lambda_{t}}\right)$ is a martingale. Then

$$
\Sigma_{t}=\Sigma_{0} e^{-\int_{0}^{t} \frac{\sigma_{z}^{2}(s)}{G s} \mathrm{~d} s}
$$

and

$$
\begin{aligned}
\mathrm{d} \sqrt{G_{t}} & =-\frac{1}{2 \sqrt{G_{t}}} \sigma_{z}^{2}(t) \mathrm{d} t+\Sigma_{t} \mathrm{~d}\left(\frac{1}{\lambda_{t}}\right) \\
& =-\frac{1}{2 \sqrt{G_{t}}} \sigma_{z}^{2}(t) \mathrm{d} t+\mathrm{d} \mathcal{M}_{t} .
\end{aligned}
$$

So,

$$
\sqrt{G_{T}}-\sqrt{G_{t}}=-\int_{t}^{T} \frac{\sigma_{z}^{2}(s)}{2 \sqrt{G_{s}}} \mathrm{~d} s+M_{T}-M_{0}
$$


and since $G_{T}=0$ because $\Sigma_{T}=0$ we have

$$
\sqrt{G_{t}}=\mathbb{E}\left(\int_{t}^{T} \frac{\sigma_{z}^{2}(s)}{2 \sqrt{G_{s}}} \mathrm{~d} s \mid \sigma_{z}^{2}(u), 0 \leq u \leq t\right) .
$$

Equation (11) in Collin-Dufresne and Fos (2016).

\subsubsection{Characterization of the equilibrium}

In this subsection we shall give necessary and sufficient conditions to guarantee that $(H, \lambda, X)$ is an equilibrium in the context of pricing rules satisfying

$$
\begin{aligned}
& \partial_{1} H+\frac{1}{2} \partial_{22} H \lambda_{t} \sigma_{Z}^{2}(t)=0, \\
& \partial_{t} \lambda_{t}+\frac{1}{2} \partial_{y y} \lambda_{t} \sigma_{Z}^{2}(t)=0 \text { a.a. } t \geq 0, y \in \mathbb{R},
\end{aligned}
$$

where $\sigma_{Z}^{2}$ is deterministic and càdlàg. Conditions (16) and (17) specify a subclass of pricing rules and thus of admissible strategies. Note that condition (16) is condition (ii) in Proposition 4 and condition (17) is (14). Observe that all the functions considered in this subsection $H, \lambda, \sigma^{2}$ are deterministic, see Remark 9 below. We shall also considered, for simplicity, that $V$ is continuous since there is not substancial difference when we introduce jumps in the fundamental value $V$. Recall that the release time $\tau$ is predictable and bounded to the insider.

From now on we shall assume the additional conditions:

(B1) $\partial_{2} H(\cdot, \xi.) \lambda_{t} \in L_{\tau}(X) \cap L_{\tau}(Z)$.

(B2) $\left(H^{-1}(t, \cdot)\left(V_{t}\right)\right)_{0 \leq t \leq \tau}, \xi_{t} \in L_{\tau}(V)$ and $\lambda\left(t, P_{t}\right) \geq C>0$.

We have the following necessary and sufficient conditions for the equilibrium.

Theorem 2 Consider an admissible triple $(H, \lambda, X)$ with $(H, \lambda)$ satisfying (16) and (17). Assume that the fundamental value $V$ is a continuous martingale. Then, necessary and sufficient conditions for an equilibrium are given by:

(i) $H\left(\tau, \xi_{\tau}\right)=V_{\tau}$

(ii) $Y_{t}, 0 \leq t<e s s \sup \tau$, is an $\mathbb{F}$-local martingale. 
Proof. Assume $(i)-(i i)$, we show that $(H, \lambda, X)$ is an equilibrium. Set now, for $t \in[0, \infty)$,

$$
I(t, y, v):=\int_{y}^{H^{-1}(t, \cdot)(v)} \frac{v-H(t, x)}{\lambda(t, H(t, x))} \mathrm{d} x .
$$

After some straightforward calculations, we have that

$$
\partial_{1} I+\frac{1}{2} \partial_{22} I \lambda_{t}^{2} \sigma_{Z}^{2}(t)=F(t, v)
$$

Where $F(t, v) \in \mathbb{C}^{1,1}$. Now, consider an admissible continuous strategy $X$, by using Itô-Wentzell's formula (see for instance Bank and Baum (2004)), we have

$$
\begin{aligned}
I\left(\tau, \xi_{\tau}, V_{\tau}\right) & =I\left(0,0, V_{0}\right)+\int_{0}^{\tau} \partial_{3} I\left(t, \xi_{t}, V_{t}\right) \mathrm{d} V_{t}+\int_{0}^{\tau} \partial_{1} I\left(t, \xi_{t}, V_{t}\right) \mathrm{d} t \\
& +\int_{0}^{\tau} \partial_{2} I\left(t, \xi_{t}, V_{t}\right) \mathrm{d} \xi_{t}+\frac{1}{2} \int_{0}^{\tau} \partial_{22} I\left(t, \xi_{t}, V_{t}\right) \mathrm{d}[\xi, \xi]_{t} \\
& +\int_{0}^{\tau} \partial_{23} I\left(t, \xi_{t}, V_{t}\right) \mathrm{d}[\xi, V]_{t}+\frac{1}{2} \int_{0}^{\tau} \partial_{33} I\left(t, \xi_{t}, V_{t}\right) \sigma_{V}^{2}(t) \mathrm{d} t
\end{aligned}
$$

By construction, $\xi_{0}=0, \mathrm{~d} \xi_{t}=\lambda_{t} \mathrm{~d} Y_{t}$. Also by (18) and the fact that $V$ and $Z$ are independent, and the admissibility conditions

$$
\partial_{23} I\left(t, \xi_{t}, V_{t}\right) \mathrm{d}[\xi, V]_{t}=-\frac{1}{\lambda_{t}} \mathrm{~d}[\xi, V]_{t}=-\mathrm{d}[X, V]_{t}=0
$$

then using (18) and (19), we get

$$
\begin{aligned}
I\left(\tau, \xi_{\tau}, V_{\tau}\right) & =I\left(0,0, V_{0}\right)+\int_{0}^{\tau} \partial_{3} I\left(t, \xi_{t}, V_{t}\right) \mathrm{d} V_{t}+\int_{0}^{\tau}\left(P_{t}-V_{t}\right)\left(\mathrm{d} X_{t}+\mathrm{d} Z_{t}\right) \\
& +\frac{1}{2} \int_{0}^{\tau} \partial_{33} I\left(t, \xi_{t}, V_{t}\right) \sigma_{V}^{2}(t) \mathrm{d} t+\int_{0}^{\tau} F\left(t, V_{t}\right) \mathrm{d} t
\end{aligned}
$$

rearranging the terms, we obtain

$$
\begin{aligned}
& \int_{0}^{\tau}\left(V_{t}-P_{t}\right) \mathrm{d} X_{t}-\left(I\left(0,0, V_{0}\right)+\frac{1}{2} \int_{0}^{\tau} \partial_{33} I\left(t, \xi_{t}, V_{t}\right) \sigma_{V}^{2}(t) \mathrm{d} t+\int_{0}^{\tau} F\left(t, V_{t}\right) \mathrm{d} t\right) \\
& =-I\left(\tau, \xi_{\tau}, V_{\tau}\right)+\int_{0}^{\tau} \partial_{3} I\left(t, \xi_{t}, V_{t}\right) \mathrm{d} V_{t}+\int_{0}^{\tau}\left(P_{t}-V_{t}\right) \mathrm{d} Z_{t} .
\end{aligned}
$$

Recall now the expected total wealth of an insider's strategy and taking the expectation in (20) we show that the maximum is achieved at $X$. For this it is important to note that $\partial_{33} I(t, y, v)$ does not depend on $y$ and thus $\partial_{33} I\left(t, \xi_{t}, V_{t}\right)$ does not depend of $\xi$. Then $I\left(0,0, V_{0}\right)+\frac{1}{2} \int_{0}^{\tau} \partial_{33} I\left(t, \xi_{t}, V_{t}\right) \sigma_{V}^{2}(t) \mathrm{d} t+\int_{0}^{\tau} F\left(t, V_{t}\right) \mathrm{d} t$ 
has the same value for any insider's strategy. The result follows from the following points.

1. The processes $\int_{0}^{\cdot} \partial_{3} I\left(t, \xi_{t}, V_{t}\right) \mathrm{d} V_{t}$ and $\int_{0}^{\cdot}\left(P_{t}-V_{t}\right) \mathrm{d} Z_{t}$ are martingales by (B2) and (A2), hence, since $\tau$ is bounded, $\int_{0}^{\tau} \partial_{3} I\left(t, \xi_{t}, V_{t}\right) \mathrm{d} V_{t}$ and $\int_{0}^{\tau}\left(P_{t}-V_{t}\right) \mathrm{d} Z_{t}$ have null expectation.

2. By $(i)$ and $(18)$ we have that $\lambda_{t} \partial_{22} I\left(\tau, \xi_{\tau}, V_{\tau}\right)=\partial_{2} H\left(\tau, \xi_{\tau}\right)>0$ and that $\lambda_{t} \partial_{2} I\left(\tau, \xi_{\tau}, V_{\tau}\right)=-V_{\tau}+$ $H\left(\tau, \xi_{\tau}\right)=0$, so we have a maximum value of $-E\left[I\left(\tau, \xi_{\tau}, V_{\tau}\right)\right]$ for our strategy $X$.

Assumption (ii) together with condition (B1) guarantee the rationality of prices, given $X$. In fact from (16)

$$
\mathrm{d} P_{t}=\lambda_{t} \partial_{2} H\left(t, \xi_{t}\right) \mathrm{d} Y_{t},
$$

so, $P$ is an $\mathbb{F}$-local martingale and, by condition (B1) it is an $\mathbb{F}$-martingale. Then from $(i)$, and on the set $\{t \leq \tau\}$ we have

$$
P_{t}=\mathbb{E}\left(H\left(\tau, \xi_{\tau}\right) \mid \mathcal{F}_{t}\right)=\mathbb{E}\left(V_{\tau} \mid \mathcal{F}_{t}\right)=\mathbb{E}\left(\mathbb{E}\left(V_{\tau} \mid \mathcal{H}_{t}\right) \mid \mathcal{F}_{t}\right)=\mathbb{E}\left(V_{t} \mid \mathcal{F}_{t}\right)
$$

If we assume that $(H, \lambda, X)$ is an equilibrium we show that $(i)-(i i)$ hold true. First note that $(i)$ is a necessary condition for equilibrium by Proposition 2. Finally, from the Itô formula, we have that

$$
\mathrm{d} Y_{t}=\frac{\mathrm{d} P_{t}}{\lambda_{t} \partial_{2} H\left(t, \xi_{t}\right)}
$$

Since prices are rational, given $X$, then we see that $(i i)$ holds true.

rule0.5em0.5em

Remark 9 In this subsection we have assumed that $\lambda(t, x)$ is a deterministic function. We could introduce some randomness in $\lambda(t, x, y)$, with the additional hypothesis $(\mathbf{H 1})$, and some integrability conditions with respect to $\mathrm{d}_{v}\left(\frac{1}{\lambda}\right)$, to conclude that if $\int_{0}^{\cdot} \mathrm{d}_{v} \frac{1}{\lambda\left(s, P_{s}, v_{s}\right)}$ is a martingale, then Theorem 2 holds.

\subsection{Case when $\tau$ is not predictable to the insider}

If $\tau$ is not predictable market is not efficient. We consider stopping times that are not predictable and independent of the rest of observable random objects $(V, P, Z, \ldots)$ that is

$$
\mathcal{H}_{t}=\overline{\mathcal{G}_{t} \vee \sigma(\tau \wedge s, 0 \leq s \leq t)}
$$


with $\mathcal{G}_{t}$ independent of $\tau$, that $\mathbb{P}(\tau>t)>0$ for all $0 \leq t<T \in \overline{\mathbb{R}}_{+}$and that $\tau$ has a density with respect to the Lebesgue measure. In the same way that in (13) we can obtain that for any $\tau$ in this class, we have also

$$
\begin{aligned}
0 & =\mathbf{1}_{[0, \tau)}(t)\left(V_{t}-P_{t}\right) \frac{\eta_{t}}{\lambda_{t}}+\frac{\eta_{t \wedge \tau}}{\lambda_{t \wedge \tau}} P_{t \wedge \tau} \\
& -\int_{0}^{t \wedge \tau} P_{s} \mathrm{~d}\left(\frac{\eta_{s}}{\lambda_{s}}\right)-\int_{0}^{t \wedge \tau} \frac{\eta_{s}}{\lambda_{s}}\left(\partial_{1} H_{t}+\frac{1}{2} \partial_{22} H_{t} \lambda_{t}^{2} \sigma_{Z}^{2}(t)\right) \mathrm{d} s+\mathcal{M}_{t} .
\end{aligned}
$$

However, now, the jump of $\mathbf{1}_{[0, \tau)}(t)$ is relevant when we do the canonical decomposition. In fact if for certain $\varrho_{t}>0$, absolutely continuous, we have that

$$
\frac{\mathbf{1}_{[0, \tau)}(t)}{\varrho_{t}}
$$

is an $\mathbb{H}$-martingale, then we obtain, by the uniqueness of the canonical decomposition, that

$$
\left(V_{t}-P_{t}\right) \frac{\mathrm{d}\left(\frac{\eta \varrho}{\lambda}\right)_{t}^{a c}}{\mathrm{~d} t}-\left(\frac{\eta \varrho}{\lambda}\right)_{t}\left(\partial_{1} H_{t}+\frac{1}{2} \partial_{22} H_{t} \lambda_{t}^{2} \sigma_{Z}^{2}(t)\right)=0
$$

In our case $\varrho_{t}:=\mathbb{P}(\tau>t)$ :

$$
\mathbb{P}\left(\tau>s \mid \mathcal{H}_{t}\right)=\mathbb{P}(\tau>s \mid \tau>t) \mathbf{1}_{[0, \tau]}(t)=\frac{\mathbb{P}(\tau>s)}{\mathbb{P}(\tau>t)} \mathbf{1}_{[0, \tau]}(t)
$$

As a consequence everything runs parallel to the predictable case if we replace $\lambda$ by $\frac{\lambda}{\varrho}$. We have the following corollaries.

Corollary 3 Assume that $(H, \lambda, X)$ is an equilibrium, then we have:

(i) $Y$ is an $\mathbb{F}$-local martingale

(ii) If $V_{t} \neq P_{t}$, a.s. on $[[0, \tau))$, then $\frac{\eta \varrho}{\lambda}$ is a positive local martingale.

Corollary 4 Assume that $(H, \lambda, X)$ is an equilibrium. In the case that $\lambda_{t}=\lambda\left(t, P_{t}\right)$ (independent of $v$ ) and $V_{t} \neq P_{t}$ a.s. (except for a set with $\mathbb{P} \otimes$ Leb zero measure) on $[[0, \tau))$ we have that

$$
\partial_{t} \lambda\left(t, P_{t}\right)+\frac{1}{2} \partial_{x x} \lambda\left(t, P_{t}\right) \lambda^{2}\left(t, P_{t}\right) \sigma_{Z}^{2}(t)-\lambda\left(t, P_{t}\right) \partial_{s} \log \varrho_{s}=0
$$

a.s. on $[[0, \tau))$.

Example 5 In Back-Baruch (2004), the authors consider the case where the admissible strategies are absolutely continuous, $\mathrm{d} Z_{t}=\sigma_{t} \mathrm{~d} B_{t}$, where $B$ is a Brownian motion, $H(t, x)=x, \lambda_{t}=\lambda\left(P_{t}\right)$ and the release time 
is random with $\tau \sim \exp (r)$ (independent of the other variables involved). In this case Equation (4) becomes

$$
\frac{1}{2} \partial_{x x} \lambda_{s} \sigma_{s}^{2}+\frac{r}{\lambda_{s}}=0
$$

that is the Equation (1.21) in Back-Baruch (2004).

We also have the following results, where for the sake of simplicity we also assume that $V$ is a continuous martingale. We use the same notation as in the previous section. First we have the following result.

Proposition 5 If an admissible triple $(H, \lambda, X)$ is an equilibrium, then

$$
V_{t}-H\left(t, \xi_{t}\right)-\frac{\lambda_{t}}{\eta_{t} \varrho_{t}} \mathbb{E}\left(\int_{t}^{T} \partial_{2} H\left(s, \xi_{s}\right) \eta_{s} \varrho_{s} \mathrm{~d} X_{s} \mid \mathcal{H}_{t}\right)=0 \text {, a.s. } \mathbb{P} \otimes \text { Leb on }[[0, \tau)),
$$

where

$$
\varrho_{t}:=\mathbb{P}(\tau>t)
$$

Proof. This a direct consequence of Theorem 1 together with the independence of $\tau$ and $\mathcal{G}_{t}$ and the (conditional) Fubini's Theorem (see for instance Theorem 1.1.7 in Applebaum (2004)). Also we use (21). rule0.5em0.5em

Hence we have analogous results to the predictable case if we replace $\lambda$ by $\frac{\lambda}{\varrho}$ and the horizon by $T$, however since $T$ can be infinite we need to assume that $\left(\frac{\eta \varrho}{\lambda}\right)_{t}$ is well defined when $t \rightarrow T$. In particular we have:

Proposition 6 Assume that $\lim _{t \uparrow T} \frac{\eta_{t} \varrho_{t}}{\lambda_{t}}$ is well defined. If an admissible triple $(H, \lambda, X)$ is an equilibrium then we have:

$$
\begin{aligned}
& \text { (i) } \lim _{t \uparrow T} H\left(t, \xi_{t}\right)=\lim _{t \uparrow T} V_{t}, \\
& \text { (ii) } \frac{\mathrm{d}\left(\frac{\eta \varrho}{\lambda}\right)_{t}^{a c}}{\mathrm{~d} t}\left(V_{t}-H_{t}\right)-\left(\frac{\eta \varrho}{\lambda}\right)_{t}\left(\partial_{1} H_{t}+\frac{1}{2} \partial_{22} H_{t} \lambda_{t}^{2} \sigma_{Z}^{2}(t)\right)=0, \\
& \text { (iii) } \partial_{1} H\left(t, \xi_{t}\right)+\frac{1}{2} \partial_{22} H\left(t, \xi_{t}\right) \lambda^{2}(t) \sigma_{Z}^{2}(t)=0, \\
& \text { a.s. on }[[0, \tau)) \text {. }
\end{aligned}
$$

And similarly we can give sufficient conditions for having an equilibrium in the context of pricing rules 
satisfying

$$
\begin{aligned}
& \partial_{1} H+\frac{1}{2} \partial_{22} H \lambda^{2} \sigma_{Z}^{2}(t)=0, \\
& \partial_{t} \lambda_{t}+\frac{1}{2} \partial_{y y} \lambda_{t} \lambda_{t}^{2} \sigma^{2}(t)-\lambda_{t} \partial_{t} \log \varrho_{s}=0 \text { a.a. } t \geq 0, y \in \mathbb{R} .
\end{aligned}
$$

We obtain a result in line with Theorem 2.

Theorem 3 Assume that the fundamental value $V$ is a continuous martingale. Then, there exist an equilibrium in the set of admissible triples $(H, \lambda, X)$ with $(H, \lambda)$ satisfying (22) and (23) for all $(t, y) \in \mathbb{R}_{+} \times \mathbb{R}$, if the following conditions hold:

(i) $\lim _{t \uparrow T} H\left(t, \xi_{t}\right)=\lim _{t \uparrow T} V_{t}$,

(ii) $Y_{t} 0 \leq t<T$, is an $\mathbb{F}$-local martingale.

\section{Concluding remarks}

In this paper we elucidates the behaviour of an equilibrium in the market when a particular asset is traded in presence of an insider who knows its fundamental price and tries to maximizes her portfolio. We assume, following Kyle (1985), that the market price of this asset is fixed by the total aggregate demand and that it obeys a very general pricing rule, by allowing the instantaneous impact of the demand, known as price pressure, to be random. We consider also a random release time, a time when the fundamental value of the asset becomes public. Situation changes according to the predictability or not of this release time. The framework we consider encompasses the particular models discussed in the literature and the results give the conditions one has to check to find an equilibrium in the particular model.

\section{References}

[1] Applebaum, D. (2009). Lévy processes and stochastic calculus. Cambridge University Press.

[2] Aase, K.K., Bjuland, T., Øksendal, B. (2012a) Strategic insider trading equilibrium: A filter theory approach. Afrika Matematika 23 (2), 145-162.

[3] Aase, K.K., Bjuland, T., Øksendal, B. (2012b) Partially informed noise traders. Mathematics and Financial Economics 6, 93-104 
[4] Amendinger, J., Imkeller, P., Schweizer, M. (1998) Additional logarithmic utility of an insider. Stochast. Proc. Appl. 75, 263-286.

[5] Back, K. (1992) Insider trading in continuous time. The Review of Financial Studies, 5 (3), 387-409.

[6] Back, K. (1993) Asymmetric information and options. The Review of Financial Studies, Vol. 6 No. $3: 435-472$.

[7] Back, K., Baruch, S. (2004). Information in securities markets: Kyle meets Glosten and Milgrom. Econometrica, 72(2), 433-465.

[8] Back, K., Pedersen, H. (1998) Long-lived information and intraday patterns. Journal of Financial Markets 1, 385-402.

[9] Bank, P., Baum, D. (2004) Hedging and portfolio optimization in financial markets with a large trader. Mathematical Finance, 14(1), 1-18.

[10] Biagini, F. and Øskendal, B. (2005) A general stochastic calculus approach to insider trading. App. Math. \& Optim. 52, 167-181.

[11] Biagini, F. and Øskendal, B. (2006) Minimal variance hedging for insider trading. International Journal of Theoretical \& Applied Finance 9, 1351-1375.

[12] Biagini, F., Hu, Y., Meyer-Brandis, T. Øksendal, B. (2012) Insider trading equilibrium in a market with memory. Mathematics and Financial Economics 6, 229-247.

[13] Caldentey R., Stacchetti, E. (2010) Insider trading with a random deadline. Econometrica, Vol. 78, No. $1,245-283$.

[14] Campi, L., Çetin, U. (2007) Insider trading in an equilibrium model with default: a passage from reduced-form to structural modelling. Finance and Stochastics, Vol. 4:591-602.

[15] Campi, L., Çetin, U., Danilova, A. (2013) Equilibrium model with default and dynamic insider information. Finance and Stochastics. 17(3), 565-585.

[16] Cho, K. (2003). Continuous auctions and insider trading: uniqueness and risk aversion. Finance and Stochastics, Vol. 7:47-71.

[17] Collin-Dufresne, P., Fos, V. (2016). Insider trading, stochastic liquidity, and equilibrium prices. Econometrica, 84(4), 1441-1475. 
[18] Corcuera, J.M., Imkeller, P., Kohatsu-Higa, A., Nualart, D. (2004) Additional utility of insiders with imperfect dynamical information. Finance and Stochastics, 8, 437-450.

[19] Corcuera, J. M., Farkas, G., Di Nunno, G., and Øksendal, B. (2010). Kyle-Back's model with Lévy noise. Preprint series. Pure mathematics http://urn. nb. no/URN: NBN: no-8076.

[20] Corcuera, J.M., Di Nunno, J. (2018) Kyle-Back's model with a random horizon. J. Theor. Appl. Finan. 21, 1850016 . https://doi.org/10.1142/S0219024918500164.

[21] Cuoco, D., and Cvitanić, J. (1998) Optimal consumption choices for a 'large'investor. Journal of Economic Dynamics and Control, 22(3), 401-436.

[22] Danilova, A. (2010) Stock market insider trading in continuous time with imperfect dynamic information. Stochastics: an international journal of probability and stochastic processes, 82 (1), 111-131.

[23] Di Nunno, G., Pamen, O.M., Øksendal, B., and Proske, F. (2011) A general maximum principle for anticipative stochastic control and application to insider trading. In Advanced Mathematical Methods for Finance Eds. G. Di Nunno and B. Øksendal. pages 181-221.

[24] Di Nunno, G., Kohatsu-Higa, A., Meyer-Brandis, T., Øksendal, B., Proske, F., and Sulem, A. (2008) Anticipative stochastic control for Lévy processes with application to insider trading. Mathematical Modelling and Numerical Methods in Finance. Handbook of Numerical Analysis, Bensoussan and Zhang (eds.). North Holland.

[25] Di Nunno, G., Meyer-Brandis, T., Øksendal, B., and Proske, F. (2006) Optimal portfolio for an insider in a market driven by Lévy processes. Quantitative Finance 6 (1), 83-94.

[26] Draouil, O. and Øksendal, B. (2016) Optimal insider control and semimartingale decomposition under enlargement of filtration. Stochastic Analysis and Applications, 34, 1045-1056.

[27] Ernst, P.A., Rogers, L.C.G., and Zhou, Q. (2017) The value of foresight. Stochastic Processes and their Applications, 127, 3913-3927.

[28] Grorud, A. and Pontier, M. (1998) Insider trading in a continuous time market model. International Journal of Theoretical and Applied Finance, 1(03), 331-347.

[29] Grorud, A. and Pontier, M. (2001). Asymmetrical information and incomplete markets. International Journal of Theoretical and Applied Finance, 4(02), 285-302.

[30] Grorud, A. and Pontier, M. (2005) Financial market model with influential informed investors. International Journal of Theoretical and Applied Finance, 8(06), 693-716. 
[31] Imkeller, P., Pontier, M., Weisz, F. (2001) Free lunch an arbitrage possibilities in a financial market with an insider. Stochast. Proc. Appl. 92, 103-130.

[32] Jacod, J. and Shiryaev, A.N. (1987) Limit Theorems for Stochatic Processes. Springer-Verlag. Berlin.

[33] Karatzas, I. Pikovski, I. (1996) Anticipative portfolio optimization. Avd. Appl. Prob. 28, 1095-1122.

[34] Kohatsu-Higa, A. (2007) Models for insider trading with finite utility. Paris-Princeton Lectures on Mathematical Finance Series: Lect. Notes in Maths, 1919, 103-172. Springer-Verlag. Berlin.

[35] Kyle, A. S. (1985) Continuous auctions and insider trading. Econometrica, Vol. 53 No. 6:1315-1335.

[36] Lasserre, G. (2004) Asymmetric information and imperfect competition in a continuous time multivariate security model. Finance and Stochastics, Vol. 8, No. 2:285-309.

[37] Liptser, Robert S.; Shiryaev, Albert N. (2001) Statistics of Random Processes II. Applications. Translated from the 1974 Russian original by A. B. Aries. Second, revised and expanded edition. Applications of Mathematics (New York), 6. Stochastic Modelling and Applied Probability. Springer-Verlag, Berlin.

[38] Malkiel, B. G. (2007) A random walk down Wall Street: the time-tested strategy for successful investing. WW Norton \& Company.

[39] Revuz, D. and Yor, M. (1999) Continuous Martingales and Brownian motion. 3rd edition. SpringerVerlag, New York. 